\title{
Endometriosis and Assisted Reproductive Technologies: Maximizing Outcomes
}

\author{
Eric S. Surrey, MD ${ }^{1}$ \\ ${ }^{1}$ Colorado Center for Reproductive Medicine, Lone Tree, Colorado \\ Semin Reprod Med 2013;31:154-163
}

\begin{abstract}
Address for correspondence and reprint requests Eric S. Surrey, MD, 10290 Ridgegate Circle, Lone Tree, CO 80124

(e-mail: esurrey@colocrm.com).
\end{abstract}

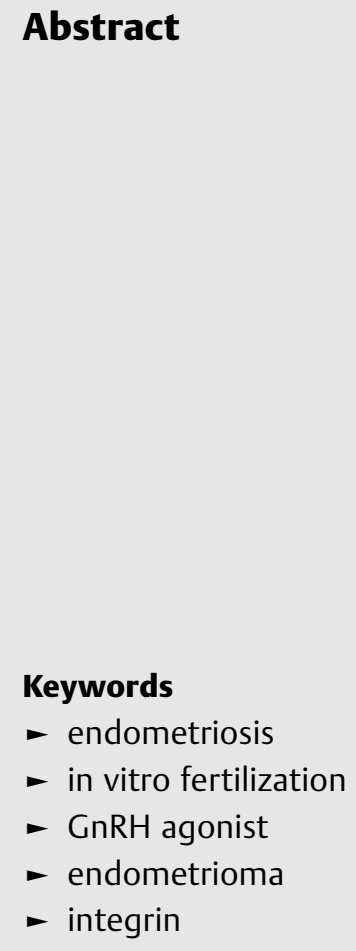

In vitro fertilization (IVF) represents the most efficient means of overcoming endometriosis-related infertility. Compromised pelvic anatomy and a hostile peritoneal environment are bypassed. Despite the results of early trials, more contemporary outcomes data would suggest that when controlled for age, IVF cycle outcome is not compromised by the presence of endometriosis. One exception to this concept is the finding that patients with ovarian endometriomas demonstrate poorer response to gonadotropin therapy, although it is not clear that this affects the likelihood of implantation. Surgical ablation of superficial endometriosis has no clear impact on IVF pregnancy rates, although a small number of recent trials suggest that pre-cycle resection of deeply infiltrative disease may be beneficial. With the exception of traditional gynecologic indications, there is no evidence to suggest that resection of ovarian endometriomas has any positive impact on cycle outcome. There are, in fact, data demonstrating that resection may exert a deleterious effect on ovarian reserve. A subset of patients will benefit from administration of a prolonged course of a gonadotropin-releasing hormone agonist prior to an IVF cycle. However, the characteristics of that subset have not been identified. It would be logical to consider this approach in women with more advanced disease, severe symptoms, and a history of implantation failure. Data on the impact of other pre-cycle medical interventions such as aromatase inhibitors, danazol, or oral contraceptives are more limited. There is also no evidence to suggest that the ovarian stimulation associated with IVF induces progression of endometriosis.
The relationships between endometriosis and infertility and possible etiologies have been previously discussed. Although surgical management can overcome anatomical distortion caused by this disease, it would be unlikely that such intervention would have an appreciable effect on the alterations in cytokine concentrations, gene expression, or other inflammatory processes that might impede conception in patients with endometriosis. However, in vitro fertilization (IVF)-embryo transfer should not only bypass abnormal pelvic anatomy but also remove gametes from an otherwise hostile peritoneal environment. The 2010 Society for Assisted Reproductive Technology (SART) Clinic Summary reported that 3777 fresh IVF cycles with a primary indication of endometriosis using nondonor oocytes were initiated in the United States during that year. ${ }^{1}$ This represented only $3.9 \%$ of the 95,625 total cycles. Given the relatively common occurrence of endometriosis in infertile women, this rather low percentage may reflect the lack of performance of routine diagnostic laparoscopy, the inclusion of minimal endometriosis under the category of "unexplained infertility," or the inclusion of endometriosis patients under other primary diagnoses, which were believed to be of greater significance, any one of which would result in an underrepresentation of this diagnosis.

In this review, I address the impact, if any, of endometriosis on IVF outcome and whether this impact can be altered by surgical or medical interventions. Lastly, the question of whether ovarian stimulation associated with IVF can have an impact on disease progression is discussed.
Issue Theme Ramifications and Adaptations to Endometriosis-Induced Infertility; Guest Editor, Dan I. Lebovic, MD
Copyright @ 2013 by Thieme Medical Publishers, Inc., 333 Seventh Avenue, New York, NY 10001, USA. Tel: +1(212) 584-4662.
DOI http://dx.doi.org/ 10.1055/s-0032-1333481. ISSN 1526-8004. 


\section{Does Endometriosis Affect IVF Outcome?}

A controversial issue is whether endometriosis per se exerts a deleterious effect on IVF outcomes. If the primary effect of endometriosis on infertility is a fundamental effect on oocyte quality or implantation, then IVF would not be expected to have a benefit. Several early studies implied that fertilization, implantation, and pregnancy rates in endometriosis patients were significantly compromised in comparison with controls. $^{2-5}$ It is important to note that in these trials, outcomes were compromised in control groups as well. In contrast, Olivennes and colleagues reported a $30 \%$ delivery rate per embryo transfer in 360 IVF cycles performed on 214 endometriosis patients in contrast to a $37.5 \%$ rate in 166 cycles performed on 111 controls with tubal disease, a difference that was not statistically significant. ${ }^{6}$ Others have confirmed these findings. ${ }^{7,8}$

Barnhart and coworkers performed a meta-analysis addressing this issue and included 27 trials published from 1983 to $1998 .{ }^{9}$ The authors concluded that the chance of conceiving from IVF was significantly lower for endometriosis patients than for tubal factor controls (odds ratio [OR]: 0.56; 95\% confidence interval $[\mathrm{CI}]: 0.44$ to 0.70 ). They also reported that endometriosis patients experienced significantly lower fertilization and implantation rates with a lower number of oocytes obtained. Once again, mean implantation and pregnancy rates were low in both groups (12.72\% versus $18.08 \%$ ). Note that these outcome statistics do not generally reflect current practice.

A more recent large retrospective analysis concluded that live-birth rates were similar for patients with endometriosis and tubal factor infertility (66.0\% versus $66.7 \%) .{ }^{10}$ Both groups had poorer outcomes than those with unexplained infertility (78.8\%). Nevertheless, implantation rates, a more accurate reflection of IVF outcome, were similar among all three groups. According to the 2010 SART registry, agematched patients with endometriosis fared no differently than the overall population of women undergoing IVF (-Table 1).

\section{Does Endometriosis Severity Affect IVF Outcome?}

If the overall population of endometriosis patients fare as well as controls in more recent analyses of IVF outcomes, then does this hold true with regard to varying degrees of disease severity? It has been suggested that advanced stage endometriosis may induce dysfunctional granulosa cell estrogen and progesterone receptor expression. ${ }^{11}$

Earlier trials had reported significantly lower pregnancy rates after IVF in patients with more advanced disease. ${ }^{12,13}$ However, it is important to note that in these studies, oocytes were obtained by laparoscopic as opposed to by transvaginal ultrasound-guided techniques. Dense pelvic adhesions and ovarian disease may have significantly limited the ability to aspirate oocytes effectively in patients with more severe disease, thus compromising outcome. Using ultrasoundguided oocyte aspiration, Azem et al noted reduced fertilization, pregnancy, and birth rates per cycle in 58 patients with stages III and IV endometriosis in comparison with 60 controls with tubal factor infertility. ${ }^{14}$ Unfortunately, no comparisons were made with patients with less extensive disease, and, in addition, delivery rates were low in both of the groups (6.7\% versus $16.6 \%$, respectively). Pal and coworkers reported that although fertilization rates were significantly lower in patients with stage III and IV in comparison with stage I and II endometriosis, implantation, clinical pregnancy, and miscarriage rates were similar between the groups. ${ }^{15}$ Several large investigations have demonstrated no relationship between disease severity and ongoing pregnancy or miscarriage rates. $^{6,7}$

As part of the previously described meta-analysis, Barnhart et al also compared outcomes in patients previously diagnosed with stage I/II endometriosis to those with stage III/IV disease. ${ }^{9}$ Women with severe disease were noted to have significantly lower peak estradiol levels and number of oocytes retrieved as well as implantation and pregnancy rates than those with mild endometriosis.

More recently, Kuivasaari et al reported that despite a significantly younger mean age, implantation rates were lower for patients with stage III/IV endometriosis as opposed to either those with stage I/II disease or a control group with tubal infertility ${ }^{16}$ (- Table 2).

Ballester and coworkers reported that patients with deeply infiltrative endometriosis, which would not typically be captured with standard scoring systems, have significantly lower pregnancy rates than endometriosis patients with more superficial lesions (58\% versus $83 \% ; p=0.003$ ). ${ }^{17}$ These investigators created a nomogram predicting pregnancy rates

Table 1 Endometriosis and In Vitro Fertilization: 2010 SART Registry ${ }^{a}$

\begin{tabular}{|l|l|l|l|l|}
\hline Age (y) & $<35$ & $35-37$ & $38-40$ & $41-42$ \\
\hline Live birth/cycle (\%) & 41.6 & 33.1 & 24.8 & 14.0 \\
\hline Endometriosis & 41.7 & 31.9 & 22.1 & 12.5 \\
\hline All diagnoses & \multicolumn{4}{|l|}{} \\
\hline Implantation rate (\%) & 36.7 & 26.4 & 18.0 & 11.3 \\
\hline Endometriosis & 36.9 & 27.0 & 17.7 & 9.6 \\
\hline All diagnoses
\end{tabular}

aModified from 2010 Society for Assisted Reproductive Technology (SART) Clinic Summary Report. ${ }^{1}$ 
Table 2 Effect of Endometriosis Stage on In Vitro Fertilization/Intracytoplasmic Sperm Injection Outcomes ${ }^{\text {a }}$

\begin{tabular}{|l|l|l|l|}
\hline \multirow{2}{*}{} & \multicolumn{2}{|c|}{ Endometriosis stage } & \multirow{2}{*}{ Tubal factor } \\
\cline { 2 - 3 } & $\mathrm{I} / \mathrm{II}$ & $\mathrm{III} / \mathrm{IV}$ & \\
\hline Patients & 31 & 67 & 87 \\
\hline Cycles & 58 & 150 & 184 \\
\hline Age, y & $32.6 \pm 4.4$ & $30.8 \pm 4.8^{*}$ & $33.7 \pm 4.3$ \\
\hline Implantation rate (\%) & 28.3 & $13.7^{*}$ & 22.1 \\
\hline
\end{tabular}

${ }^{*} p<0.05$.

a Modified from Kuivasaari et al. ${ }^{16}$

including such parameters as age, serum antimüllerian hormone (AMH) level, and number of cycles and reported that the presence of deeply infiltrative disease was the strongest predictor of clinical pregnancy.

\section{Are Outcomes Affected by the Presence of an Endometrioma?}

Although the presence of ovarian endometriotic cysts (endometriomas) should perhaps be addressed as an independent factor, it is difficult to truly assess the effect of these lesions on IVF outcome in isolation given that most of the patients with these lesions are likely to have concomitant peritoneal disease that could have an independent effect. The effect of endometrioma size per se has also not been evaluated as an independent variable.

Yanushpolsky et al reported a higher incidence of pregnancy loss, a decreased number of oocytes retrieved, as well as an adverse effect on embryo quality in endometrioma patients. ${ }^{18}$ In contrast, Olivennes et al demonstrated no impact of endometriomas on any outcome parameter. ${ }^{6} \mathrm{Sev}-$ eral investigators described a decrease in ovarian response requiring the use of higher gonadotropin doses in patients with such lesions. ${ }^{19,20}$ However, cumulative pregnancy and live-birth rates were unaffected.

Somigliana and colleagues reported that this effect was more marked in those patients with multiple and/or larger cysts. ${ }^{20}$ In a more recent article, the same group compared the response of each ovary to gonadotropin stimulation in women with a unilateral endometrioma and noted that the development of follicles with a mean diameter $>15 \mathrm{~mm}$ on the day of human chorionic gonadotropin administration was similar between the two sides. ${ }^{21}$ This finding was confirmed by others. ${ }^{22}$

In a recent retrospective series, Ballester and coworkers reported that the total number of endometriomas, size of the largest lesions, and the presence of unilateral or bilateral lesions had no impact on cycle outcome. ${ }^{23}$ However, the presence of concomitant deeply infiltrating disease also had a significant and deleterious effect on the cumulative likelihood of pregnancy. Serum anti-AMH level also was highly predictive, which would emphasize the importance of completing a thorough evaluation of ovarian reserve (as well at the rest of a thorough infertility evaluation) before initiating therapy.

\section{Does Surgical Management of Nonovarian Endometriosis Improve IVF Outcome?}

The effectiveness of surgical ablation or resection of endometriotic implants as the sole treatment of endometriosisrelated subfertility has been addressed elsewhere. The question of whether such intervention in the absence of ovarian endometriomata would enhance IVF cycle outcome has been less extensively evaluated. One prospective randomized trial reported that, although laparoscopic carbon dioxide laser ablation of endometriosis at the time of gamete intrafallopian transfer (GIFT) had no effect on cycle outcome, pregnancy rates in subsequent cycles of patients who failed to conceive were significantly higher than in controls with endometriosis who underwent GIFT alone. ${ }^{24}$ Surrey and Schoolcraft reported that controlled ovarian hyperstimulation and IVF cycle outcomes were similar between two groups of patients with endometriosis but without endometriomas, one of which had undergone surgical resection within 6 months and the other had undergone surgical resection $>6$ months to 5 years prior to oocyte aspiration (ongoing pregnancy rates $63.6 \%$ versus $60.53 \%$, respectively). ${ }^{25}$ (-Fig. 1) Regression analysis revealed no impact of either the time interval between surgery and oocyte aspiration or endometriosis score on implantation rates. Bedaiwy et al confirmed this finding. ${ }^{26}$ It would appear that the previously described benefit derived from such surgery in enhancing spontaneous conception may be masked by the greater impact on implantation and pregnancy achieved with the assisted reproductive technologies.

Two more recent studies would appear to suggest that surgical management may improve cycle outcomes in certain circumstances. A Norwegian retrospective trial compared IVF outcomes in patients with stage I/II endometriosis who either underwent complete surgical resection of lesions or diagnostic laparoscopy only. ${ }^{27}$ Implantation (30.9\% versus $23.9 \%$; $p=0.02)$ and live-birth (27.7\% versus $20.6 \% ; p=0.04)$ rates were significantly higher in the patients who underwent surgical intervention. A second trial evaluated two groups of patients with "symptoms and/or signs" of deeply invasive endometriosis who elected to undergo extensive surgical resection prior to IVF or to proceed directly to IVF. ${ }^{28}$ Patients who underwent surgery required significantly higher gonadotropin doses resulting in a lower number of oocytes retrieved, but implantation $(32.1 \pm 30.6 \%$ versus $19 \pm 25.1 \%$; $p=0.03$ ) and overall pregnancy rates (41\% versus $24 \%$; 


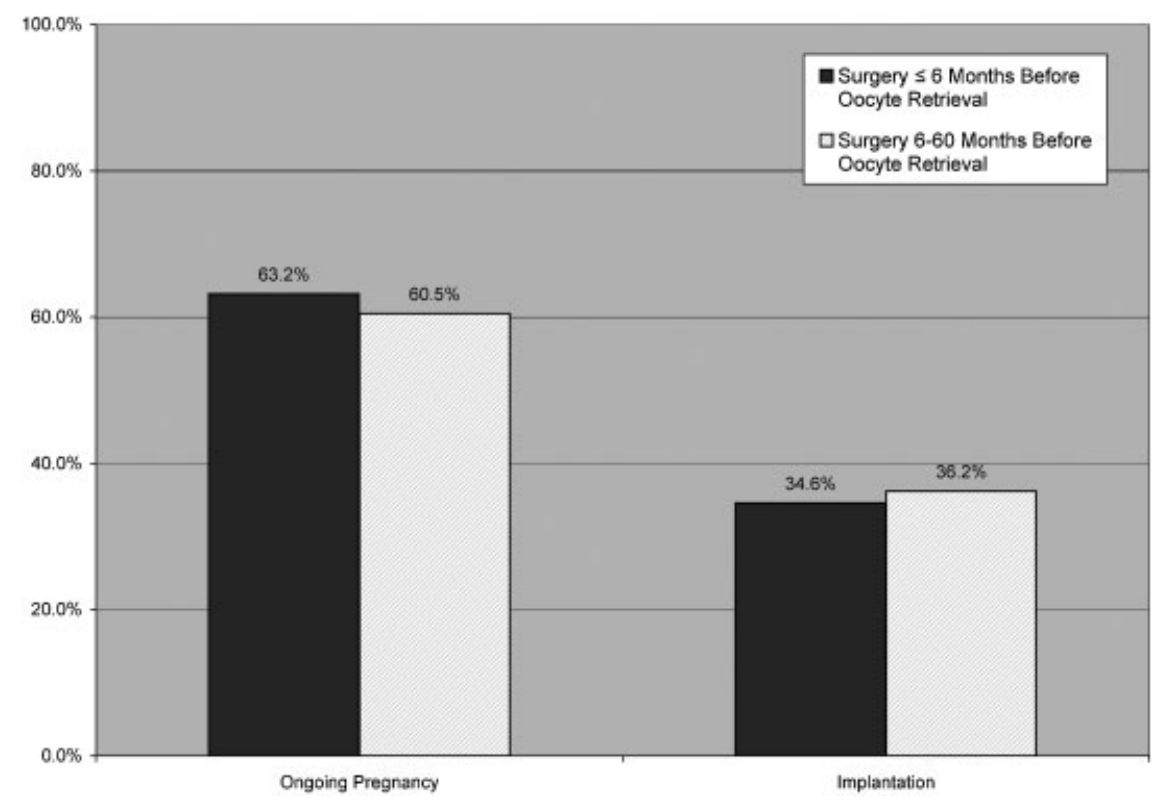

Figure 1 Ongoing pregnancy and implantation rates in patients undergoing surgical resection of nonovarian endometriosis either $\leq 6$ months or 6 to 60 months prior to oocyte aspiration and in vitro fertilization. Modified from Surrey and Schoolcraft (Fig. 1). ${ }^{25}$

$p=0.004)$ were significantly higher. The design of both of these studies is subject to selection bias. An additional weakness of the latter trial is the lack of definitive diagnosis of endometriosis in all patients. Thus one cannot draw definitive conclusions. In an analysis of patients with all stages of endometriosis who failed an initial IVF cycle and then underwent surgical resection prior to a second cycle, no differences in day 3 embryo quality were appreciated. ${ }^{29}$ The need for appropriately designed prospective randomized trials to address this issue are critical before one can state that surgical intervention prior to IVF is of benefit in any specific patient population.

\section{Does Pre-Cycle Resection of Endometriomas Affect IVF Outcomes?}

The question of whether resection of endometriomas either enhances or has a deleterious effect on IVF cycle outcome is addressed in detail elsewhere. However, two recent reviews of the literature are helpful. Tsoumpo et al published a metaanalysis of the effect of surgical treatment of endometriomas or expectant management on subsequent IVF cycles. ${ }^{30}$ Metaanalysis was performed on 5 of 20 eligible studies. These authors noted no significant differences in pregnancy rates or gonadotropin responses between the groups, suggesting little benefit in surgical intervention. In a more recent Cochrane Database review, Benschop and coworkers confirmed a lack of evidence of any benefit from either aspiration or cystectomy compared with expectant management with regard to clinical pregnancy rates or number of mature oocytes retrieved. ${ }^{31}$ Cystectomy was associated with a decreased response to controlled ovarian hyperstimulation in comparison with expectant management.

One of the presumed benefits of endometrioma resection was purported to be the avoidance of inadvertent exposure of oocytes to endometrioma fluid at the time of aspiration. However, at least one group of investigators has shown that such exposure has no impact on fertilization or early embryo development rates. ${ }^{32}$ Nevertheless, it does make sense to make every effort to avoid entering an endometrioma during oocyte retrieval procedures to prevent peritoneal leak of contents.

If resection of endometriomas prior to IVF is generally not beneficial, then can this intervention cause harm? Several investigators have shown that the response to gonadotropins of operated versus nonoperated ovaries was significantly reduced after unilateral cystectomy. ${ }^{33-36}$ Somigliana et al calculated that this corresponded to a $53 \%$ reduction in response (95\% CI, 35 to 72 ) that was not affected by the size of the cyst excised. ${ }^{34}$ In fact, this same group reported that, of 93 women who underwent pre-cycle surgery for unilateral endometriomas, an absence of follicular growth in the operated but not the contralateral ovary occurred in $13 \%$ of cases. ${ }^{37}$ Others have failed to show such a deleterious effect, however. ${ }^{38-40}$

Given the lack of convincing evidence supporting benefit of routine resection and potential surgical risk as well as damage to ovarian function, one would ask if there are any indications for removing an endometrioma prior to an IVF cycle. GarciaVelasco and Somigliana recently published an elegant opinion article that addresses this issue. ${ }^{41}$ They claimed that it would be reasonable to consider surgical intervention in patients who have never previously undergone laparoscopy to confirm the diagnosis of endometriosis, those with progressive pain, those masses that exhibit rapid growth and/or have suspicious ultrasound features, those of a significant enough size to create concern for rupture in pregnancy, and an inability to access the remainder of the ovary. Others should be managed expectantly ( - Table 3). However, when surgical intervention is undertaken, it is critical to use meticulous 
Table 3 Proposed Indications for Pre-In Vitro Fertilization Cycle Endometrioma Resection ${ }^{\mathrm{a}}$

\begin{tabular}{|l|}
\hline - No prior surgical confirmation of endometriosis \\
\hline - Severe pelvic pain attributable to mass \\
\hline - Rapid growth \\
\hline - Suspicious sonographic features \\
\hline - Compromised access to remaining follicles \\
\hline - Concern for rupture in pregnancy due to size \\
\hline
\end{tabular}

${ }^{a}$ Modified from Garcia-Velasco and Somigliana. ${ }^{41}$

techniques with a goal of carefully avoiding compromise of ovarian blood supply and destroying otherwise healthy normal tissue.

\section{Does Pre-IVF Cycle Medical Suppression Improve Outcomes?}

Traditional medical therapy for symptomatic endometriosis such as progestins, danazol, and gonadotropin-stimulating hormone $(\mathrm{GnRH})$ agonists has been shown to have little impact on enhancing spontaneous pregnancy rates in infertile endometriosis patients. ${ }^{42}$ However, if the negative effect of this disease process on fertility returns rapidly after discontinuation of medication, then one could hypothesize that any benefits of medical suppression on enhancing fertility would be most evident if pregnancy could be achieved during a time of maximal suppression. This could only occur with the use of the assisted reproductive technologies.

Most of the investigations in this regard have examined prolonged use of GnRH agonists prior to IVF. In a prospective randomized multicenter trial, Surrey et al evaluated the effect of a 3-month course of a GnRH agonist administered immediately prior to initiating controlled ovarian hyperstimulation $(\mathrm{COH})$ in preparation for IVF in 25 patients with surgically confirmed endometriosis. ${ }^{43}$ Significantly higher ongoing pregnancy rates with a trend toward higher implantation rates were appreciated in comparison to controls with endometriosis who underwent standard $\mathrm{COH}$ protocols and IVF without prolonged GnRH agonist therapy. Of note is the fact that a higher percentage of patients who received prolonged agonist therapy had more advanced disease, a group that one would expect to have inherently poorer outcomes (-Fig. 2).

These findings have been demonstrated by others. Seven previous studies of varying design have assessed the effect of suppression with a GnRH agonist (GnRHa) before IVF or GIFT. $^{44-50}$ The length of suppression varied from 6 weeks to 7 months. Some studies lacked control groups, but a beneficial effect of pretreatment was suggested by all.

Rickes and colleagues evaluated the effect of pre-cycle surgical treatment of endometriosis alone or in combination with a 6-month postoperative treatment course of a GnRHa on IVF or $\mathrm{COH}$-intrauterine insemination (IUI) outcome in a prospective randomized trial of 110 patients. ${ }^{51}$ The pregnancy rates were significantly higher for both forms of fertility therapy in those patients treated with a prolonged postoperative GnRHa course. However, when patients were stratified based on disease stage, a statistically significant difference was only appreciated among patients with stages III/IV endometriosis who underwent IVF. A summary of the results of the randomized trials is displayed in -Table 4.

Sallam et al more recently performed a Cochrane Database analysis of three of these prospective randomized trials including 163 endometriosis patients undergoing 3 to 6 months of pre-cycle GnRHa treatment. ${ }^{52}$ This intervention

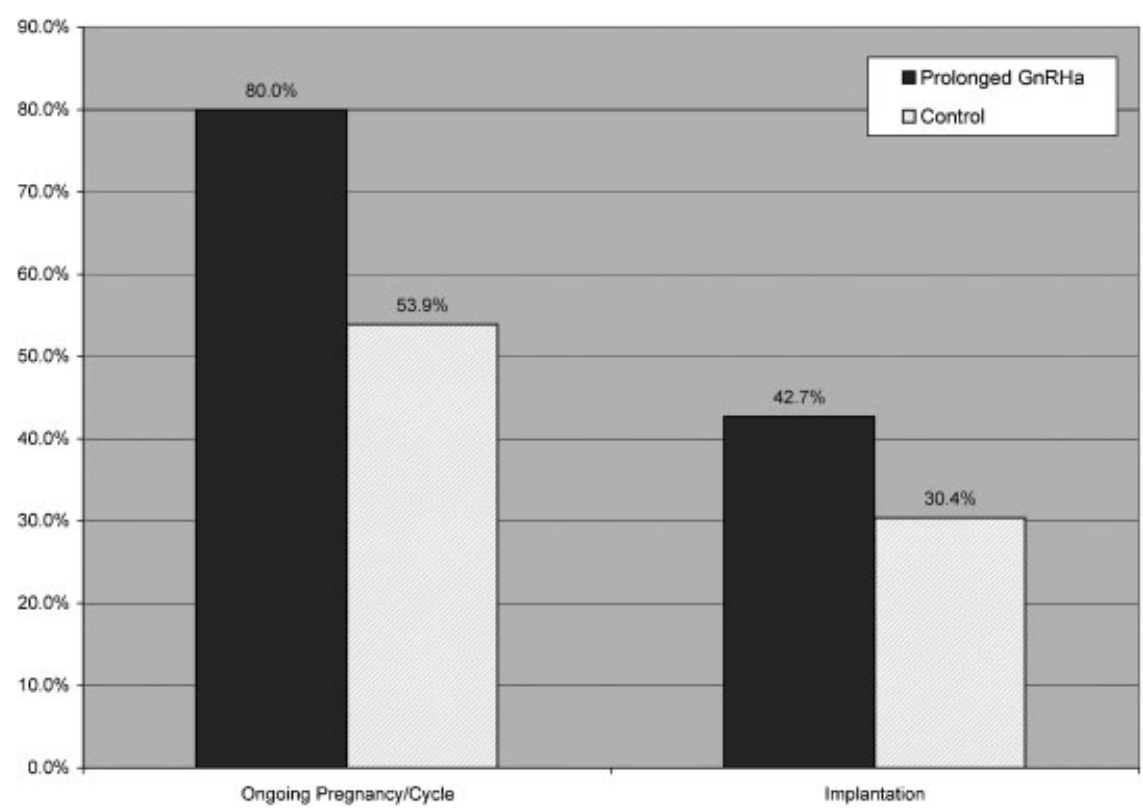

Figure 2 Ongoing pregnancy and implantation rates in endometriosis patients after a 3-month course of gonadotropin-releasing hormone agonist $(\mathrm{GnRHa})$ versus control prior to in vitro fertilization. Modified from Surrey et al. ${ }^{43}$ (Fig. 1). ${ }^{*} p<0.05$. 
Table 4 In Vitro Fertilization and Endometriosis: Prolonged Pre-Cycle Gonadotropin-Releasing Hormone Agonist

\begin{tabular}{|c|c|c|c|c|c|c|}
\hline \multirow{3}{*}{$\begin{array}{l}\text { Study } \\
{\text { Chedid et } \mathrm{al}^{44}}^{44}\end{array}$} & \multirow{3}{*}{$\begin{array}{l}\text { Design } \\
\text { Retrospective }\end{array}$} & \multirow{3}{*}{$\begin{array}{l}\text { Patients/Cycles } \\
145 / 174\end{array}$} & \multicolumn{4}{|c|}{ Clinical Pregnancy Rates (\%) } \\
\hline & & & \multirow{2}{*}{$\begin{array}{l}\text { No GnRHa } \\
23^{*}\end{array}$} & \multirow{2}{*}{$\begin{array}{l}\text { Standard } \\
\text { GnRHa } \\
39\end{array}$} & \multicolumn{2}{|c|}{$\begin{array}{l}\text { Prolonged GnRHa } \\
\text { (duration) }\end{array}$} \\
\hline & & & & & 46 & $(3 \mathrm{mo})$ \\
\hline Nakamura et $a^{50}$ & Retrospective & $32 / 32$ & - & $27^{*}$ & 67 & $(126 \pm 57) d$ \\
\hline Marcus and Edwards ${ }^{49}$ & "Semi-randomized" & $84 / 181$ & - & 11 & 35 & $(2-7 \mathrm{mo})$ \\
\hline Remorgida et al ${ }^{46}$ & Prospective randomized & 60 & 33 & 32 & 56 & $(6 \mathrm{mo})$ \\
\hline Dicker et $\mathrm{al}^{48}$ & Prospective randomized & 64 & $5^{*}$ & - & 33 & $(6 \mathrm{mo})$ \\
\hline Surrey et $\mathrm{al}^{43}$ & Prospective randomized & $51 / 51$ & - & $56.5^{*}$ & 78.3 & (3 mo) \\
\hline \multirow[t]{3}{*}{ Rickes et $a^{51}$} & $\begin{array}{l}\text { Postoperative prospective } \\
\text { randomized }\end{array}$ & $47 / 82$ & & 47 & 75 & (6 mo) \\
\hline & & & Stage I/II & 50 & 56 & \\
\hline & & & Stage III/IV & $40^{*}$ & 82 & \\
\hline
\end{tabular}

GnRHa, gonadotropin-releasing hormone agonist.

${ }^{*} p<0.05$ versus prolonged GnRHa.

resulted in significantly improved rates of both live birth (OR: 9.1\%; 95\% CI, 1.08 to 78.22) and clinical pregnancy (OR: 4.28; $95 \% \mathrm{CI}, 2.0$ to 9.15$)$.

There are no studies that compare varying lengths of suppressive therapy or whether this approach should be offered to specific subgroups of endometriosis patients given the associated increased expense and time delay before pregnancy can occur.

The mechanism of action of this effect has not been clearly established. It has been suggested that, aside from their primary mechanism, GnRHa may act to diminish concentrations of peritoneal fluid metalloproteinase tissue inhibitors, downregulate peritoneal fluid inflammatory proteins, and increase apoptosis and expression of pro-apoptotic proteins. ${ }^{53-55}$ Others have shown that GnRHa may significantly decrease endometrial nitric oxide synthase expression. ${ }^{56}$

A great deal of interest has surrounded the role of endometrial $\beta 3$ integrin expression in this setting. Lessey et al previously demonstrated that administration of a GnRHa for 3 months to women with stage I/II endometriosis and aberrant endometrial $\beta 3$ integrin expression resulted in a $64 \%$ rate of return of expression. ${ }^{57}$ Ruan and coworkers reported that, in a murine model, impaired endometrial $\beta 3$ integrin and leukemia-inhibitory factor expression as well as uterine receptivity resulting from ovarian stimulation were partially restored after GnRHa administration. ${ }^{58}$

Given that the pregnancy rate in control patients who were not administered prolonged agonist therapy still remained relatively high in the trial by Surrey et al, it is clear that not all endometriosis patients require this intervention. ${ }^{43}$ These data beg the question of whether endometrial $\beta 3$ integrin expression can be used as a marker to determine which patients might be candidates for prolonged pre-cycle GnRHa therapy. In a case-control study of 74 consecutive IVF candidates believed to be at high risk for implantation defects due to prior IVF failure despite adequate embryo quality and/ or endometriosis, we reported a $48.6 \%$ prevalence of absent endometrial integrin expression. ${ }^{59}$ Of those who had undergone laparoscopy, 52.8\% had a diagnosis of endometriosis of whom 57.1\% had stage III/IV disease. Miller and coworkers reported that live-birth rates from IVF were significantly increased in patients with positive versus negative integrin expression (38\% versus 7\%; $p<0.05$ ). ${ }^{60}$ Farrell and colleagues reported a small series of 11 patients with aberrant integrin expression and in phase endometrial biopsies who were administered an 8-week course of a GnRH agonist in conjunction with norethisterone acetate prior to IVF. Nine experienced ongoing pregnancies. ${ }^{61}$

In an effort to resolve this issue, Surrey and colleagues recently reported the results of a prospective randomized pilot trial of 36 endometriosis patients undergoing IVF. ${ }^{62}$ The patients were randomized after assessing $\beta 3$ integrin expression to receive either 3 months of GnRHa prior to initiating ovarian stimulation or to proceed directly to ovarian stimulation. Interestingly, a trend toward higher pregnancy rates that did not achieve statistical significance was noted in integrin-positive patients administered prolonged GnRHa. This is the opposite of what one would have predicted. In this study, the value of a negative biopsy in predicting ongoing IVF pregnancy after integrin expression was only 44.4\% (-Fig. 3). These results would either suggest that evaluating integrin expression is of little value in determining which patients would benefit from prolonged GnRHa, or they may have been confounded by a limited sample size or by the fact that patients in the control groups moved directly to IVF after biopsy, whereas study group patients did not undergo stimulation for 3 months. There are data suggesting that the performance of an endometrial biopsy alone may enhance implantation rates as a result of the localized injury, particularly in patients with a history of implantation failure. ${ }^{63,64}$

There are a host of other unresolved issues. We do not know the ideal duration of therapy. Is there a need for 


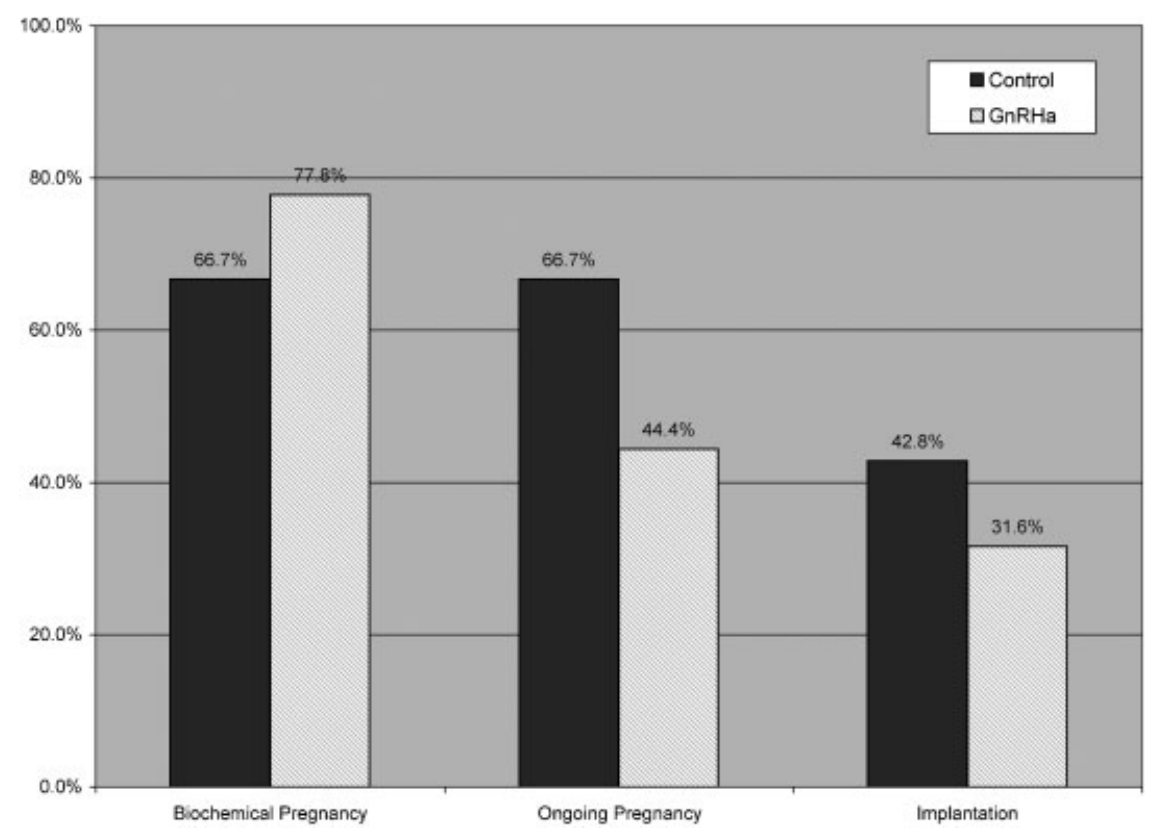

Figure 3 In vitro fertilization cycle outcomes after randomization of endometriosis patients with negative pre-cycle endometrial $\beta 3$ integrin expression to 3 months of gonadotropin-releasing hormone agonist $(\mathrm{GnRHa})$ or no therapy prior to controlled ovarian hyperstimulation and oocyte aspiration. No differences were statistically significant. Modified from Surrey et al ${ }^{62}$ (Fig. 3).

repeated courses of therapy if an initial cycle is unsuccessful? A significant concern with the administration of GnRHa to patients with compromised or diminished ovarian reserve is the deleterious effect on ovarian response to subsequent gonadotropin therapy. In this circumstance, it may be wise to vitrify all embryos after appropriate stimulation and then administer the GnRHa prior to endometrial preparation for frozen embryo transfer.

There is even more limited data regarding the use of other suppressive medical therapies. Tei et al reported nine patients with reduced endometrial integrin expression. Repeated IVF failures who were treated with a 12-week course of danazol $400 \mathrm{mg}$ showed a significant increase in expression in the first ovulatory cycle after completion of therapy. ${ }^{65}$ No clinical outcomes were reported, however.

More recently, Miller and colleagues reported that the administration of the aromatase inhibitor letrozole $5 \mathrm{mg}$ daily for days 2 to 6 of gonadotropin stimulation resulted in cycle outcomes that were similar to patients who were integrin receptor positive and not treated with this agent. ${ }^{60}$ The weakness of this retrospective trial is the fact that the authors did not randomize integrin-negative patients to similar protocols with or without the use of letrozole. Although the results are encouraging, it is difficult to draw definitive conclusions.

De Ziegler and coinvestigators recently evaluated the role of a 6- to 8-week course of oral contraceptives in patients planning IVF with either surgically diagnosed endometriosis or those with sonographic suspicion of the presence of endometriosis. ${ }^{66}$ The use of oral contraceptives resulted in higher pregnancy rates per retrieval than in controls $35 \%$ versus $12.9 \%, p=0.01$ ). This impact was even greater in those with presumed endometriomas. It is important to note that this was a retrospective trial and that control patients were both significantly older and had higher baseline folliclestimulating hormone levels. An additional concern is the lack of documentation of endometriosis in all patients.

The dearth of appropriately designed randomized trials makes it difficult to determine which subset of endometriosis patients would benefit from pre-IVF cycle GnRHa (or potentially other suppressive therapy). I would propose that suppressive intervention be considered in the subsets of infertile endometriosis patients with significantly advanced disease, those with severe pain, and/or those with a history of prior IVF cycle failure particularly after transfer of good quality embryos.

\section{Does IVF Have an Impact on Endometriosis?}

Most of this article has been devoted to a discussion of whether endometriosis and its treatments have an impact on IVF outcome. It is perhaps appropriate to also ask whether IVF and $\mathrm{COH}$ have an impact on the progression of endometriosis given the resulting supraphysiologic estradiol levels that could theoretically stimulate disease progression. There is a limited amount of data in this regard, but the results are reassuring.

D'Hooghe et al evaluated 67 patients with stage III/IV endometriosis who underwent ovarian stimulation after surgery for either IVF and/or IUI. ${ }^{67}$ Cumulative disease recurrence as calculated by life table analysis was lower after stimulation for IVF than for IUI cycles despite exposure to significantly higher circulating estradiol levels. Similarly, Benaglia and colleagues noted no worsening in endometriosis symptom scores or change in size of either endometriomas or peritoneal nodules evaluated by serial transvaginal ultrasound examinations in the 3 to 6 months after an IVF cycle. ${ }^{68}$ The authors reported that $22 \%$ reported improvement in 
symptoms, whereas $11 \%$ reported worsening of symptoms during this follow-up period.

\section{Conclusions}

Although IVF clearly represents the most efficient way for infertile endometriosis patients to conceive, less invasive alternatives should not be ignored. Taken as a whole, patients with endometriosis should expect similar age-based outcomes from IVF as other patients. The possible exception to this contention may be the individual with advanced ovarian disease who may require more aggressive stimulation resulting in a more compromised number of oocytes and embryos.

There is inconclusive evidence to suggest that pre-cycle surgical ablation of superficial endometriosis is of benefit in enhancing IVF outcome, although the outcomes are more encouraging from a small number of studies with weaknesses in design specifically addressing the effects of resecting deeply infiltrative disease. The impact of endometriomas on IVF outcome is not overcome by resection. The indications for doing so should be limited to patients without prior diagnosis of endometriosis, those with symptoms directly related to the mass, rapidly growing lesions particularly with suspicious features, and masses that significantly limit safe access to normal ovarian tissue for oocyte aspiration. Care must be taken to preserve ovarian blood supply and normal ovarian tissue if endometrioma resection is considered to minimize any iatrogenic impact on ovarian reserve.

The administration of a prolonged course of GnRHa, and possibly other suppressive agents, appears to improve IVF cycle outcome. However, the ideal subset of endometriosis patients who are candidates for this approach has not been adequately defined. Nevertheless, primary attention might be given to those with more severe disease with severe pain and/ or a history of implantation failure.

It goes without saying that patients with endometriosis who are IVF candidates should undergo the same thorough pre-cycle evaluation as any other patient. This should include at minimum an assessment of ovarian reserve, tubal patency, the uterine cavity, and sperm function. In this way, appropriate pre-cycle therapy, ovarian stimulation protocols, and laboratory techniques can be planned to maximize a successful outcome.

\section{References}

1 Society for Assisted Reproductive Technology. 2010 Clinic summary report. Available at: www.sartcorsonline.com

2 Bergendal A, Naffah S, Nagy C, Bergqvist A, Sjöblom P, Hillensjö T. Outcome of IVF in patients with endometriosis in comparison with tubal-factor infertility. J Assist Reprod Genet 1998;15(9):530-534

3 Arici A, Oral E, Bukulmez O, Duleba A, Olive DL, Jones EE. The effect of endometriosis on implantation: results from the Yale University in vitro fertilization and embryo transfer program. Fertil Steril 1996;65(3):603-607

4 Wardle PG, Mitchell JD, McLaughlin EA, Ray BD, McDermott A, Hull MG. Endometriosis and ovulatory disorder: reduced fertilisation in vitro compared with tubal and unexplained infertility. Lancet 1985;2(8449):236-239
5 Simón C, Gutiérrez A, Vidal A, et al. Outcome of patients with endometriosis in assisted reproduction: results from in-vitro fertilization and oocyte donation. Hum Reprod 1994;9(4): 725-729

6 Olivennes F, Feldberg D, Liu HC, Cohen J, Moy F, Rosenwaks Z. Endometriosis: a stage by stage analysis-the role of in vitro fertilization. Fertil Steril 1995;64(2):392-398

7 Geber S, Paraschos T, Atkinson G, Margara R, Winston RM. Results of IVF in patients with endometriosis: the severity of the disease does not affect outcome, or the incidence of miscarriage. Hum Reprod 1995;10(6):1507-1511

8 Hickman TN; Data from the Wilford Hall Medical Center IVF-ET Program. Impact of endometriosis on implantation. J Reprod Med 2002;47(10):801-808

9 Barnhart K, Dunsmoor-Su R, Coutifaris C. Effect of endometriosis on in vitro fertilization. Fertil Steril 2002;77(6):1148-1155

10 Omland AK, Abyholm T, Fedorcsák P, et al. Pregnancy outcome after IVF and ICSI in unexplained, endometriosis-associated and tubal factor infertility. Hum Reprod 2005;20(3):722-727

11 Karita M, Yamashita Y, Hayashi A, et al. Does advanced-stage endometriosis affect the gene expression of estrogen and progesterone receptors in granulosa cells? Fertil Steril 2011;95(3): 889-894

12 Matson PL, Yovich JL. The treatment of infertility associated with endometriosis by in vitro fertilization. Fertil Steril 1986;46 (3):432-434

13 Chillik CF, Acosta AA, Garcia JE, et al. The role of in vitro fertilization in infertile patients with endometriosis. Fertil Steril 1985; 44(1):56-61

14 Azem F, Lessing JB, Geva E, et al. Patients with stages III and IV endometriosis have a poorer outcome of in vitro fertilizationembryo transfer than patients with tubal infertility. Fertil Steril 1999;72(6):1107-1109

15 Pal L, Shifren JL, Isaacson KB, Chang Y, Leykin L, Toth TL. Impact of varying stages of endometriosis on the outcome of in vitro fertilization-embryo transfer. J Assist Reprod Genet 1998;15(1): 27-31

16 Kuivasaari P, Hippeläinen M, Anttila M, Heinonen S. Effect of endometriosis on IVF/ICSI outcome: stage III/IV endometriosis worsens cumulative pregnancy and live-born rates. Hum Reprod 2005;20(11):3130-3135

17 Ballester M, Oppenheimer A, d'Argent EM, et al. Nomogram to predict pregnancy rate after ICSI-IVF cycle in patients with endometriosis. Hum Reprod 2012;27(2):451-456

18 Yanushpolsky EH, Best CL, Jackson KV, Clarke RN, Barbieri RL, Hornstein MD. Effects of endometriomas on oocyte quality, embryo quality, and pregnancy rates in in vitro fertilization cycles: a prospective, case-controlled study. J Assist Reprod Genet 1998; 15(4):193-197

19 Al-Azemi M, Bernal AL, Steele J, Gramsbergen I, Barlow D, Kennedy S. Ovarian response to repeated controlled stimulation in in-vitro fertilization cycles in patients with ovarian endometriosis. Hum Reprod 2000;15(1):72-75

20 Somigliana E, Infantino M, Benedetti F, Arnoldi M, Calanna G, Ragni $\mathrm{G}$. The presence of ovarian endometriomas is associated with a reduced responsiveness to gonadotropins. Fertil Steril 2006; 86(1):192-196

21 Benaglia L, Pasin R, Somigliana E, Vercellini P, Ragni G, Fedele L. Unoperated ovarian endometriomas and responsiveness to hyperstimulation. Hum Reprod 2011;26(6):1356-1361

22 Almog B, Shehata F, Sheizaf B, Tan SL, Tulandi T. Effects of ovarian endometrioma on the number of oocytes retrieved for in vitro fertilization. Fertil Steril 2011;95(2):525-527

23 Ballester M, Oppenheimer A, Mathieu d'Argent E, et al. Deep infiltrating endometriosis is a determinant factor of cumulative pregnancy rate after intracytoplasmic sperm injection/in vitro fertilization cycles in patients with endometriomas. Fertil Steril 2012;97(2):367-372 
24 Surrey MW, Hill DL. Treatment of endometriosis by carbon dioxide laser during gamete intrafallopian transfer. J Am Coll Surg 1994;179(4):440-442

25 Surrey ES, Schoolcraft WB. Does surgical management of endometriosis within 6 months of an in vitro fertilization-embryo transfer cycle improve outcome? J Assist Reprod Genet 2003;20 (9):365-370

26 Bedaiwy MA, Falcone T, Katz E, Goldberg JM, Assad R, Thornton J. Association between time from endometriosis surgery and outcome of in vitro fertilization cycles. J Reprod Med 2008;53(3): 161-165

27 Opøien HK, Fedorcsak P, Byholm T, Tanbo T. Complete surgical removal of minimal and mild endometriosis improves outcome of subsequent IVF/ICSI treatment. Reprod Biomed Online 2011; 23(3):389-395

28 Bianchi PH, Pereira RM, Zanatta A, Alegretti JR, Motta EL, Serafini PC. Extensive excision of deep infiltrative endometriosis before in vitro fertilization significantly improves pregnancy rates. J Minim Invasive Gynecol 2009;16(2):174-180

29 Shahine LK, Burney RO, Behr B, Milki AA, Westphal LM, Lathi RB. Embryo quality before and after surgical treatment of endometriosis in infertile patients. J Assist Reprod Genet 2009;26(2-3): 69-73

30 Tsoumpou I, Kyrgiou M, Gelbaya TA, Nardo LG. The effect of surgical treatment for endometrioma on in vitro fertilization outcomes: a systematic review and meta-analysis. Fertil Steril 2009;92(1): 75-87

31 Benschop L, Farquhar C, van der Poel N, Heineman MJ. Interventions for women with endometrioma prior to assisted reproductive technology. Cochrane Database Syst Rev 2010;(11):CD008571

32 Khamsi F, Yavas Y, Lacanna IC, Roberge S, Endman M, Wong JC. Exposure of human oocytes to endometrioma fluid does not alter fertilization or early embryo development. J Assist Reprod Genet 2001;18(2):106-109

33 Almog B, Sheizaf B, Shalom-Paz E, Shehata F, Al-Talib A, Tulandi T. Effects of excision of ovarian endometrioma on the antral follicle count and collected oocytes for in vitro fertilization. Fertil Steril 2010;94(6):2340-2342

34 Somigliana E, Ragni G, Benedetti F, Borroni R, Vegetti W, Crosignani PG. Does laparoscopic excision of endometriotic ovarian cysts significantly affect ovarian reserve? Insights from IVF cycles. Hum Reprod 2003;18(11):2450-2453

35 Esinler I, Bozdag G, Aybar F, Bayar U, Yarali H. Outcome of in vitro fertilization/intracytoplasmic sperm injection after laparoscopic cystectomy for endometriomas. Fertil Steril 2006;85(6): $1730-1735$

36 Ho HY, Lee RK, Hwu YM, Lin MH, Su JT, Tsai YC. Poor response of ovaries with endometrioma previously treated with cystectomy to controlled ovarian hyperstimulation. J Assist Reprod Genet 2002;19(11):507-511

37 Benaglia L, Somigliana E, Vighi V, Ragni G, Vercellini P, Fedele L. Rate of severe ovarian damage following surgery for endometriomas. Hum Reprod 2010;25(3):678-682

38 Alborzi S, Ravanbakhsh R, Parsanezhad ME, Alborzi M, Alborzi S, Dehbashi S. A comparison of follicular response of ovaries to ovulation induction after laparoscopic ovarian cystectomy or fenestration and coagulation versus normal ovaries in patients with endometrioma. Fertil Steril 2007;88(2):507-509

39 Marconi G, Vilela M, Quintana R, Sueldo C. Laparoscopic ovarian cystectomy of endometriomas does not affect the ovarian response to gonadotropin stimulation. Fertil Steril 2002;78(4): 876-878

40 Donnez J, Wyns C, Nisolle M. Does ovarian surgery for endometriomas impair the ovarian response to gonadotropin? Fertil Steril 2001;76(4):662-665

41 Garcia-Velasco JA, Somigliana E. Management of endometriomas in women requiring IVF: to touch or not to touch. Hum Reprod 2009;24(3):496-501
42 Hughes EG, Fedorkow DM, Collins JA. A quantitative overview of controlled trials in endometriosis-associated infertility. Fertil Steril 1993;59(5):963-970

43 Surrey ES, Silverberg KM, Surrey MW, et al. The effect of prolonged GnRH agonist therapy on in vitro fertilization-embryo transfer cycle outcome in endometriosis patients: a multicenter randomized trial. Fertil Steril 2002;78(4):699-704

44 Chedid S, Camus M, Smitz J, Van Steirteghem AC, Devroey P. Comparison among different ovarian stimulation regimens for assisted procreation procedures in patients with endometriosis. Hum Reprod 1995;10(9):2406-2411

45 Wardle PG, Foster PA, Mitchell JD, et al. Endometriosis and IVF: effect of prior therapy. Lancet 1986;1(8475):276-277

46 Remorgida V, Anserini P, Croce S, Costa M, Ferraiolo A, Capitanio GL. Comparison of different ovarian stimulation protocols for gamete intrafallopian transfer in patients with minimal and mild endometriosis. Fertil Steril 1990;53(6):1060-1063

47 Curtis P, Jackson A, Bernard A, Shaw RW. Pretreatment with gonadotrophin releasing hormone $(\mathrm{GnRH})$ analogue prior to in vitro fertilisation for patients with endometriosis. Eur J Obstet Gynecol Reprod Biol 1993;52(3):211-216

48 Dicker D, Goldman GA, Ashkenazi J, Feldberg D, Voliovitz I, Goldman JA. The value of pre-treatment with gonadotrophin releasing hormone $(\mathrm{GnRH})$ analogue in IVF-ET therapy of severe endometriosis. Hum Reprod 1990;5(4):418-420

49 Marcus SF, Edwards RG. High rates of pregnancy after long-term down-regulation of women with severe endometriosis. Am J Obstet Gynecol 1994;171(3):812-817

50 Nakamura K, Oosawa M, Kondou I, et al. Menotropin stimulation after prolonged gonadotropin releasing hormone agonist pretreatment for in vitro fertilization in patients with endometriosis. J Assist Reprod Genet 1992;9(2):113-117

51 Rickes D, Nickel I, Kropf S, Kleinstein J. Increased pregnancy rates after ultralong postoperative therapy with gonadotropin-releasing hormone analogs in patients with endometriosis. Fertil Steril 2002;78(4):757-762

52 Sallam HN, Garcia-Velasco JA, Dias S, Arici A. Long-term pituitary down-regulation before in vitro fertilization (IVF) for women with endometriosis. Cochrane Database Syst Rev 2006;(1):CD004635

53 Sharpe-Timms KL, Keisler LW, McIntush EW, Keisler DH. Tissue inhibitor of metalloproteinase- 1 concentrations are attenuated in peritoneal fluid and sera of women with endometriosis and restored in sera by gonadotropin-releasing hormone agonist therapy. Fertil Steril 1998;69(6):1128-1134

54 Ferrero S, Gillott DJ, Remorgida V, Anserini P, Ragni N, Grudzinskas JG. GnRH analogue remarkably down-regulates inflammatory proteins in peritoneal fluid proteome of women with endometriosis. J Reprod Med 2009;54(4):223-231

55 Bilotas M, Barañao RI, Buquet R, Sueldo C, Tesone M, Meresman G. Effect of GnRH analogues on apoptosis and expression of $\mathrm{Bcl}-2$, Bax, Fas and FasL proteins in endometrial epithelial cell cultures from patients with endometriosis and controls. Hum Reprod 2007;22(3):644-653

56 Wang J, Zhou F, Dong M, Wu R, Qian Y. Prolonged gonadotropinreleasing hormone agonist therapy reduced expression of nitric oxide synthase in the endometrium of women with endometriosis and infertility. Fertil Steril 2006;85(4):1037-1044

57 Lessey BA. Medical management of endometriosis and infertility. Fertil Steril 2000;73(6):1089-1096

58 Ruan HC, Zhu XM, Luo Q et al. Ovarian stimulation with GnRH agonist, but not GnRH antagonist, partially restores the expression of endometrial integrin beta ${ }_{3}$ and leukaemia-inhibitory factor and improves uterine receptivity in mice. Hum Reprod 2006; 21(10):2521-2529

59 Surrey ES, Minjarez DA, Schoolcraft WB. The incidence of aberrant endometrial alphavbeta(3) vitronectin expression in a high risk infertility population: could prolonged $\mathrm{GnRH}$ agonist therapy play a role? J Assist Reprod Genet 2007;24(11):553-556 
60 Miller PB, Parnell BA, Bushnell G, et al. Endometrial receptivity defects during IVF cycles with and without letrozole. Hum Reprod 2012;27(3):881-888

61 Farrell R, Gray D, Gindlesperger V, et al. Treatment of $\alpha v \beta_{3}$ integrin abnormalities increases pregnancy success rates after failing IVF cycles. Fertil Steril 2003;80(Suppl 3):S58

62 Surrey ES, Lietz AK, Gustofson RL, Minjarez DA, Schoolcraft WB. Does endometrial integrin expression in endometriosis patients predict enhanced in vitro fertilization cycle outcomes after prolonged GnRH agonist therapy? Fertil Steril 2010;93(2):646-651

63 Barash A, Dekel N, Fieldust S, Segal I, Schechtman E, Granot I. Local injury to the endometrium doubles the incidence of successful pregnancies in patients undergoing in vitro fertilization. Fertil Steril 2003;79(6):1317-1322

64 Raziel A, Schachter M, Strassburger D, Bern O, Ron-El R, Friedler S. Favorable influence of local injury to the endometrium in intra- cytoplasmic sperm injection patients with high-order implantation failure. Fertil Steril 2007;87(1):198-201

65 Tei C, Maruyama T, Kuji N, Miyazaki T, Mikami M, Yoshimura Y. Reduced expression of alphavbeta3 integrin in the endometrium of unexplained infertility patients with recurrent IVF-ET failures: improvement by danazol treatment. J Assist Reprod Genet 2003; 20(1):13-20

66 de Ziegler D, Gayet V, Aubriot FX, et al. Use of oral contraceptives in women with endometriosis before assisted reproduction treatment improves outcomes. Fertil Steril 2010;94(7):2796-2799

67 D'Hooghe TM, Denys B, Spiessens C, Meuleman C, Debrock S. Is the endometriosis recurrence rate increased after ovarian hyperstimulation? Fertil Steril 2006;86(2):283-290

68 Benaglia L, Somigliana E, Santi G, Scarduelli C, Ragni G, Fedele L. IVF and endometriosis-related symptom progression: insights from a prospective study. Hum Reprod 2011;26(9):2368-2372 